\title{
Médiévales
}

Langues, Textes, Histoire

48 | printemps 2005

Princes et princesses à la fin du Moyen Âge

\section{Locus et conuentus : un état des « lieux » franciscains chez Salimbene de Adam}

\section{Gisèle Besson}

\section{(2) OpenEdition}

1 Journals

Édition électronique

URL : https://journals.openedition.org/medievales/844

DOI : 10.4000/medievales.844

ISSN : $1777-5892$

Éditeur

Presses universitaires de Vincennes

\section{Édition imprimée}

Date de publication : 1 juin 2005

Pagination : 123-140

ISBN : 2-84292-169-0

ISSN : 0751-2708

\section{Référence électronique}

Gisèle Besson, «Locus et conuentus : un état des « lieux » franciscains chez Salimbene de Adam », Médiévales [En ligne], 48 | printemps 2005, mis en ligne le 02 mars 2007, consulté le 24 avril 2022.

URL : http://journals.openedition.org/medievales/844; DOI : https://doi.org/10.4000/medievales.844

Ce document a été généré automatiquement le 24 avril 2022.

Tous droits réservés 


\title{
Locus et conuentus : un état des « lieux » franciscains chez Salimbene de Adam
}

\author{
Gisèle Besson
}

1 Le mot français de "couvent» entre dans la langue au XII ${ }^{\mathrm{e}}$ ou au XIII ${ }^{\mathrm{e}}$ siècle, selon les indications des dictionnaires ${ }^{1}$. Son étymologie latine est évidente et pourtant le parallélisme apparent des deux mots n'est qu'une illusion. En effet conuentus est clairement, d'après son origine tirée de cum+uenio, un terme qui désigne d'abord un rassemblement de personnes, et ce dans tous les domaines («attroupement» dans la vie quotidienne, "assemblée des grands" dans la vie politique, "assemblée des fidèles », « synode » dans la vie religieuse...) ; l'étymologie du mot reste immédiatement perceptible en latin². De façon secondaire, le terme a entre autre été employé, par spécialisation de sens, pour désigner une communauté de religieux (ou religieuses). Puis, par métonymie, il a désigné le lieu de vie de cette communauté, les bâtiments conventuels.

2 En sens inverse, le français dérivé couvent - anciennement convent -, dont le sens reste toujours lié (directement ou par allusion ironique) au monde religieux, désigne d'abord le lieu, «la maison où des religieux et des religieuses vivent en commun » et ensuite «l'ensemble des religieux ou des religieuses qui composent la communauté $»^{3}$.

Dans la perspective d'une traduction ${ }^{4}$ de la Chronique de Salimbene de Adam, un franciscain $\mathrm{du} \mathrm{XIII}^{\mathrm{e}}$, s'est tout naturellement posée la question du vocabulaire utilisé pour désigner les établissements franciscains : l'incertitude la plus immédiate est celle des domaines d'emploi des deux termes les plus fréquents, locus et conuentus, même s'il n'y a pas de problème fondamental de sens. Pour le mot locus ${ }^{5}$, traditionnel chez les franciscains, il n'est pas facile de trouver un équivalent simple en français et bien souvent les historiens de l'Ordre conservent le mot latin. Ceux qui veulent traduire utilisent plusieurs solutions, variables selon les auteurs, et un même traducteur a souvent recours à des traductions qui varient au gré des phrases sans raison particulière : on trouve ainsi « lieux » (pour rester fidèle au latin), "établissements ", 
«local/emplacement $»^{6}$, « résidence $»^{7}$, parfois aussi une ellipse du mot dans la traduction de groupe où il figure ${ }^{8}$, voire très couramment " couvent ", ce qui est par ailleurs la traduction habituelle de conuentus.

4 La Chronique de Salimbene permet-elle d'éclairer un peu l'évolution sémantique de conuentus au XIII ${ }^{\mathrm{e}}$ siècle? Où en sont à ce moment les sens de conuentus, quel est l'éventail des emplois du terme chez ce franciscain, quelle est la place du sens dérivé, désignant les bâtiments, par rapport au sens premier de "communauté »? Une deuxième question, liée à la première, est de juger de l'usage attesté chez Salimbene par rapport à la terminologie officielle franciscaine de son époque ${ }^{9}$.

5 Il sera nécessaire de faire d'abord le point sur la terminologie en usage chez les franciscains, en rappelant très rapidement des choses connues, avant de regarder de plus près les emplois de conuentus chez Salimbene. Une confrontation avec quelques emplois de locus, mot que nous aurons déjà rencontré chemin faisant, viendra ensuite confirmer les premiers résultats obtenus.

L'évolution du vocabulaire franciscain

6 À l'origine, chez saint François, on ne rencontre aucun mot précis ${ }^{10}$ pour désigner l'installation des frères puisque par définition ils ne doivent rien posséder de particulier : on connaît assez l'inquiétude que manifestait le saint à l'idée que les frères puissent s'installer dans une construction faite pour leur usage. Les termes employés pour les ordres religieux existants, cloître ou monastère (claustrum, monasterium), n'étaient évidemment pas adaptés aux principes et au mode de vie de l'Ordre naissant. Il n'est donc question, en termes vagues et souvent complétés par des mots à valeur généralisante, que des «lieux ", des " endroits" où les frères trouvent un logement: locus a là son sens ordinaire en latin ${ }^{11}$. De la même façon, domus est employé avec son sens normal de «maison » emprunté au vocabulaire quotidien, puisque les franciscains doivent être hébergés dans des habitations banales ${ }^{12}$. Il n'y a dans toute l'œuvre de François aucun emploi de conuentus, dans aucun des sens du terme.

7 On voit que les deux mots locus et domus ont, comme plus tard conuentus, un point de départ situé dans le vocabulaire courant, non spécialisé et même éloigné de toute connotation religieuse : ils seront de bons modèles pour la spécialisation du terme qui va jouer ensuite un rôle prépondérant et donner en français le mot de couvent.

8 Cependant la situation de l'Ordre évolue rapidement: dès 1230, la bulle Quo elongati dispense les frères Mineurs de l'obligation d'observer strictement le Testament de saint François, ce qui leur ouvre la possibilité d'acquérir des locaux plus importants. En 1250, la bulle Cum tamquam ueri accorde le titre d'église conventuelle à toutes les résidences franciscaines où il y avait un nombre suffisant de frères pour "assurer le service divin » et «tenir chapitre $»^{13}$. Enfin, élément essentiel pour la question que je pose ici, les statuts du chapitre général de Narbonne en 1260 donnent une définition officielle du terme de conuentus ${ }^{14}$ : le mot sera réservé aux établissements comptant en permanence treize frères ou davantage ${ }^{15}$.

9 Si l'on consulte les historiens de l'Ordre, ils ne semblent guère s'être intéressés à cette question de terminologie : les indications sur les mots employés sont généralement peu précises et donnent une impression que ne confirmera pas l'examen de la Chronique de Salimbene. Ainsi le P. Gratien de Paris ${ }^{16}$ signale-t-il l'abandon progressif des loca, " pauvres maisons tombant souvent de vétusté », «trop petites " et "qui se prêtaient mal aux usages de la vie religieuse »; il date des environs de 1230 le moment où les franciscains commencent à se déplacer vers l'intérieur des villes, «mouvement [qui] 
prit toute son ampleur à partir de 1240 ", et il parle à ce moment de "fondation, transfert et agrandissement » de couvents. Il suggère ainsi pour locus un sens restreint qui ne correspond guère aux emplois attestés dans les textes de la deuxième moitié du XIII ${ }^{\mathrm{e}}$ siècle.

10 Avant d'en venir au texte de Salimbene que je veux examiner de plus près, il me parait utile de proposer des éléments de comparaison en formulant quelques remarques rapides sur d'autres écrits franciscains presque contemporains de Salimbene.

11 Ainsi avons-nous connaissance des débuts de l'Ordre en Allemagne par l'œuvre de Jourdain de Giano, Chronica seu Memorabilia : après une première tentative malheureuse (1217), les franciscains envoient en Allemagne une deuxième mission, dont fait partie Jourdain. Installé d'abord à Augsbourg en 1221, l'Ordre progresse ensuite très rapidement. La Chronique de Jourdain de Giano est écrite après coup, dans les années 1260 , soit une bonne vingtaine d'années avant Salimbene.

12 Dans cet ouvrage assez bref, on trouve une seule fois ${ }^{17}$ l'emploi de conuentus, pour désigner l'établissement franciscain de Rome (c'est donc un usage en avance sur les textes officiels), six emplois de locus, 12 emplois de domus, dont une attestation unique avec un adjectif indiquant le lieu, in domum Erfordensem ${ }^{18}$.

13 L'installation des franciscains en Angleterre, décidée en 1223, est l'objet du traité de Thomas d'Eccleston: les Frères arrivent à la fin de 1224 et l'Ordre se développe rapidement, sans grande difficulté, puisqu'il possède une dizaine de couvents en Angleterre avant 1230 et quarante-neuf vers 1256. Thomas est en Angleterre vers 1230-1232 et son Tractatus de aduentu fratrum Minorum in Angliam est écrit aux alentours de 1258-1260. Il cite deux fois le conuentus Londoniae, une fois le conuentus Romae: le terme reste encore très marginal. Il utilise par contre tout à fait habituellement locus ${ }^{19}$ ou domus ${ }^{20}$.

Les emplois de conuentus chez Salimbene : une évolution encore en cours

$14 \mathrm{Au}$ contraire des œuvres que je viens d'évoquer ${ }^{21}$, Salimbene fait un grand usage de conuentus, dont il présente au total 125 exemples $^{22}$.

15 Le terme a déjà commencé à évoluer à partir de son emploi primitif, puisque sa spécialisation est déjà acquise. Salimbene l'emploie en effet exclusivement en contexte religieux, avec une valeur qui peut être celle de "communauté religieuse" ou de "bâtiments où habite cette communauté ", mais on ne le trouve pas avec un sens autre: l'emploi général a disparu chez Salimbene, même s'il existe encore à cette époque ailleurs, alors que pour locus coexistent l'emploi spécifique et la valeur générale du mot $^{23}$. D'autre part, l'emploi est presque exclusivement réservé aux Mineurs ${ }^{24}$ : on ne trouve que cinq cas où le mot s'applique à d'autres ordres dont un peut même être éliminé de ce décompte puisqu'il concerne des Clarisses ${ }^{25}$ (en 579,35 ). Sur les quatre cas où conuentus ne s'applique pas à des franciscains, on en trouve un avec désignation géographique pour les Prêcheurs $(103,28)$, le conuentus Bononie, où je soupçonne un effet de confusion de termes à cause du lieu (on sait l'importance du studium de Bologne pour les franciscains et Salimbene cite cinq fois ce lieu, toujours sous l'appellation de conuentus) et à cause de l'anecdote racontée (il y est question d'une apparition dans l'infirmerie des Prêcheurs, mais c'est celle d'un frère Mineur). Il reste deux autres exemples pour les Prêcheurs ${ }^{26}(335,6$, au singulier; 339, 2, au pluriel), dont nous reparlerons en conclusion pour montrer pourquoi le terme est employé, et un pour des cisterciens $(907,24)$, mais il se trouve dans un document que cite Salimbene et ne 
correspond pas nécessairement à l'usage qu'il fait lui-même du terme ${ }^{27}$. Pour ces 124 exemples de conuentus sous la plume de Salimbene, on voit donc que pratiquement tous sont en relation avec les Mineurs.

Deuxième étape, le sens exact de conuentus. Parmi les emplois du mot, un tri permet de montrer que le sens étymologique est encore très présent.

Un premier groupe d'emplois, numériquement de loin le plus important, est constitué de locutions donnant une précision d'ordre géographique : elles sont formées soit avec un nom propre de lieu (locution courante du type conuentus Bononie, rares emplois du type conuentus de Hesio) soit avec un adjectif d'origine (du type conuentus Bononiensis). Sur les 124 emplois de conuentus chez Salimbene, 102 contiennent une précision géographique explicite, soit environ $82 \%$ des emplois; il faut y ajouter quelques cas (cinq au moins et vraisemblablement bien davantage ${ }^{28}$ ) où le syntagme ne contient pas lui-même la précision géographique parce qu'elle est déjà présente dans le contexte. On peut donc compter au total $86 \%$ au moins d'emplois à valeur géographique. C'est visiblement une terminologie entrée dans les habitudes, la manière la plus commode de désigner les établissements franciscain ${ }^{29}$. Mais cela ne permet pas encore de décider du sens exact de conuentus dans cet emploi : le sens de "communauté » est tout à fait possible, même si celui de «maison où habitent les religieux » a souvent été privilégié.

En laissant de côté les phrases ambiguës, il reste finalement une quinzaine de cas où conuentus n'est pas lié à une désignation géographique. Certains ne donnent aucune indication nette sur le sens qu'il faut attribuer au $\operatorname{mot}^{30}$. Mais dans six passages au moins, le contexte souligne très clairement la valeur étymologique de "communauté de personnes " par l'abondance d'indications complémentaires allant dans le même sens. Ainsi, à propos du couvent des Clarisses de Faenza, Salimbene parle du rassemblement de toute la communauté à l'étage où est installée l'église: "Toute la communauté des Dames de ce lieu, au nombre de soixante-douze, était assemblée à cet endroit $\aleph^{31}$. De même, stigmatisant les raffinements que s'accorde le ministre général frère Élie, il précise que celui-ci se faisait servir ses repas dans sa chambre et non au réfectoire avec toute la communauté, même s'il était évidemment logé à l'intérieur des bâtiments conventuels : «En outre il prenait rarement ses repas avec la communauté en même temps que les autres frères, mais toujours à part, seul dans sa chambre $»^{32}$. De même encore en 336, 20 sq., totus conuentus congregatus, où il faut lire l'ensemble du passage pour relever toutes les notations de foule ${ }^{33}$.

19 Il reste en fait seulement quatre cas où conuentus désigne à coup sûr le lieu et non la communauté d'hommes, ce qui est fort peu sur les 124 attestations du mot ${ }^{34}$ ! Trois de ces exemples seront étudiés en conclusion pour leur contenu ironique ; je cite ici un seul emploi : lorsque le ministre général Jean de Parme rappelle l'obligation de dire la messe "secundum rubricam ordinarii", il reproche à certains frères de se contenter parfois d'une messe des morts célébrée le matin dans le couvent et de négliger la messe du jour : "S'ils avaient le matin tôt dans le couvent une messe pour des morts, à certains endroits ils s'en contentaient et se dispensaient complètement de toute autre $<$ messe $>\|^{35}$.

Les preuves du passage au sens matériel de « couvent »?

20 Il est donc certain que l'évolution de conuentus vers son sens matériel est déjà connue de Salimbene, mais il montre une certaine réticence à employer cette valeur du mot. Que trouve-t-on en effet dans les passages où sont évoqués les bâtiments conventuels? Il faut d'abord constater que le chroniqueur parmesan ne propose guère de description 
matérielle d'un de ces établissements. On rencontre certes quelques termes spécifiques comme réfectoire (refectorium), chapitre, infirmerie, hôtellerie (forestaria), cellules des frères (cella)... mais ils sont assez rares et jamais construits avec le génitif de conuentus (d'ailleurs peu employé) : on a ainsi « la cloche du réfectoire » mais pas « du couvent », ou des expressions comme ad ianuam monasterii $(66,21)$, baptisterii, ecclesiarum. On ne trouve donc rien de comparable à ce qui est attesté un siècle plus tard, dans les Actus Beati Francisci et sociorum eius, qui fournit deux exemples de ante altare conuentus ${ }^{36}$ où le sens matériel me paraît sûr.

21 Une autre piste me semble être l'adjonction d'un adjectif qualificatif au terme qui désigne le couvent dans son sens matériel, ce qui reste d'ailleurs peu fréquent, sans doute à cause d'une évidente réticence que manifestent les franciscains à se glorifier de leur prospérité matérielle, trop éloignée de l'idéal de pauvreté originel ${ }^{37}$.

L'adjectif magnus peut sembler a priori ambigu puisqu'il peut qualifier le nombre des membres de la congrégation aussi bien que la taille des bâtiments. Aussi hésite-t-on d'abord pour comprendre exactement la phrase qui indique la préférence des Prêcheurs pour les grands établissements/ les grandes communautés, in magnis conuentibus habitare quam in paruis (p. 339, 2). Mais on rapprochera d'autres passages : l'allusion à l'Ordre des Prêcheurs qui... magnum locum habebat in Parma $(679,3)$, la mention in quodam magno monasterio pour des Cisterciens (p.908, 14), et surtout l'évocation du couvent franciscain de Ravenne : l'évêque de Mantoue, Martin de Parme, interroge son compatriote Salimbene sur l'endroit qu'occupent les Mineurs à Ravenne ; Salimbene désigne une magna ecclesia et magnum campanile que leur a offerts l'archevêque de Ravenne Philippe (p.629, 15-32). Il faut noter que face à l'étalage complaisant de cette richesse, Salimbene s'est contenté du mot locus pour désigner cet établissement qu'il appelle ailleurs à quatre reprises conuentus Rauenne (par exemple p.910, 25). La situation est à peu près aussi ambiguë pour l'adjectif sollemnis (remarquable, illustre), mais il ne s'applique dans le texte qu'à quatre monastères bénédictins dont Cluny et Saint-Gall (p. 306, 25).

Plus clair est le cas de pulcher, qui qualifie bien des bâtiments, comme en 313, 20 un pulcherrimum monasterium cistercien. Mais il n'est jamais employé avec conuentus. On trouvera un pulcher locus pour les Prêcheurs (p. 104, 13) et Salimbene signale la construction d'un beau réfectoire pour les Mineurs, fratres Minores de Parma fecerunt pulchrum refectorium in Prato Sancti Herculani, ubi habitant (p. 759, 1 et 2); par contre lorsque le pape Innocent fait construire sur ses domaines une église et un couvent où il souhaite héberger vingt-cinq frères (pulcrum locum fecit et pulchram ecclesiam, p. 86, 16), les Mineurs refusent parce que cela leur paraît trop beau. On commence à voir se dessiner avec ces exemples les domaines d'emploi de conuentus et locus dans des termes qui ne sont pas ceux qui apparaissaient dans les définitions habituellement données. L'étude des adjectifs se termine avec la mention de bonus, qui qualifie lui aussi nettement le bâtiment conventuel, et qu'on rencontre une fois pour un bonus conuentus des Prêcheurs (p. 335, 6), et une fois pour évoquer les deux couvents que possèdent les Mineurs à Tarascon et Beaucaire, in quolibet castro est bonus conuentus fratrum Minorum (p. 337, 11).

Remarques sur l'évolution vers le sens matériel

D'un point de vue linguistique, on peut trouver quatre éléments qui ont contribué à l'évolution sémantique du terme de conuentus. D'une part, comme je l'ai signalé dès le 
début, cette évolution n'était pas isolée dans le vocabulaire désignant ces établissements religieux, avec les spécialisations de sens qui ont affecté domus et locus.

D'autre part, la souplesse du génitif latin lui permet d'exprimer différents rapports de sens entre le nom et son complément au génitif, sans que la structure apparente subisse de variation. Ainsi les constructions fréquentes du type locus fratrum (67 ex. sur environ 140 emplois de locus au sens d'« établissement religieux», soit $47 \%$ ) présentent un banal génitif de possession : « le locus qui appartient aux frères ». Or, si l'on rencontre la locution conuentus fratrum Minorum, elle peut paraître a priori bâtie sur le même modèle, et pourtant, dans son sens premier, il n'en est rien, puisque le groupe ne signifie pas "la communauté qui appartient aux frères", mais «la communauté que constituent les frères", construction où le génitif latin a une valeur subjective ${ }^{38}$. Au demeurant cette deuxième locution est rare (trois exemples seulement, avec une indication géographique explicite dans deux cas et implicite en contexte pour un). La différence de fréquence me paraît là encore significative : locus fratrum Minorum est banal, parce que c'est un bon moyen pour spécifier le sens «technique » de locus et le différencier du sens ordinaire de "lieu, endroit»; conuentus fratrum Minorum au contraire est rare parce que relativement tautologique chez Salimbene, le terme de conuentus étant, on l'a vu, pratiquement réservé aux franciscains : il est donc inutile de préciser qu'il s'agit d'une communauté de frères Mineurs! Avec le temps et l'évolution $\mathrm{du}$ sens de conuentus vers sa signification matérielle, les deux locutions paraissent bâties sur le même modèle et leur parallélisme peut faciliter l'évolution de la seconde, puisque le changement de sens n'impose aucun changement de construction grammaticale.

Ensuite l'emploi préférentiel des groupes contenant le mot conuentus comme complément circonstanciel de lieu a pu faciliter le passage au sens matériel de ce mot : « habiter dans une communauté » peut se comprendre comme « habiter dans les lieux où loge cette communauté ». Or effectivement, une large majorité des emplois de conuentus sont des compléments circonstanciels de lieu, souvent groupés avec le verbe habito/moror et la préposition in suivie de l'ablatif (76 exemples), ou avec ire et la prép. ad suivie de l'accusatif (12), soit un peu plus de $70 \%$ des constructions où apparaît conuentus.

Enfin, et sans doute en liaison avec la remarque précédente, l'association fréquente de conuentus avec une précision d'ordre géographique amenait aisément à donner un sens local à des expressions comme conuentus de Parma ou parmensis, dont je pense, au vu de l'étude qui a été présentée, qu'elles ont bien des chances chez Salimbene d'être encore proches du sens étymologique de conuentus, et qu'elles sont en tout cas le noyau dont est partie l'évolution sémantique du $\operatorname{mot}^{39}$.

Quelques remarques sur le rôle de locus

Pour compléter cette étude et confirmer ses résultats, il est intéressant de regarder du côté des termes "concurrents » de conuentus. Je me limite ici à locus et je laisse de côté le terme de domus (79 emplois, pas tous pour les Mineurs, sur 363, ont un sens religieux) qui m'a paru moins important par rapport à mon projet, mais qui pourrait être étudié. Le rôle de locus reste prédominant par un certain nombre d'aspects qui nous montreront d'autres traces des réticences à l'apparition du nouveau sens de conuentus.

Bien loin de ne désigner que les premières et misérables installations dont se sont contentés les franciscains à la naissance de l'Ordre, le mot locus reste encore extrêmement important, il est le terme générique pour tous les établissements, quelle 
que soit leur taille; il reste le mot le plus général, pour d'autres Ordres comme pour les Mineurs ${ }^{40}$. Il est employé 140 fois environ (quelques cas sont ambigus entre sens général et sens proprement religieux), soit un peu plus que les 125 conuentus, ce qui s'explique entre autres par le fait qu'il ne s'applique pas exclusivement aux franciscains.

Il peut remplacer le terme précis d'ermitage, peu employé mais attesté chez Salimbene (eremus, eremitorium, 11 emplois), par exemple pour La Verna (uniquement désigné par locus) ou Greccio (désigné aussi comme heremitorium, p. 451 ou 801). Il est en outre le seul mot employé pour certains établissements, une quinzaine dans le total des établissements franciscains évoqués dans l'œuvre. Il peut s'agir de petites communautés, ainsi pour Fanano (Modène), cité une seule fois dans la Chronique (p. 594, 28) et si peu connu que le répertoire des établissements franciscains de J. Moorman ${ }^{41}$ semble ignorer sa présence à cette époque, puisqu'il date la fondation de "avant 1343 », ou pour Medesano (Parme) cité une fois comme établissement franciscain (p. 240, 29) et ignoré de J. Moorman ${ }^{42}$; de même pour les Prêcheurs, à Parme, Salimbene emploie le terme de locus à une époque où, précise-t-il, il n'y avait là que trois frères (p. 848, 26). Mais le terme désigne aussi des établissements importants, qui ne sont cités qu'une fois, par exemple Toulouse ou Salins, où l'on sait pourtant qu'un couvent a été fondé en 1230 par le comte de Châlons, et où, selon Salimbene lui-même, se trouvent vingt-deux frères (p. 754, 11), ce qui ne correspond pas du tout à la définition officielle donnée à Narbonne en 1260. Peut-être s'agit-il dans certains cas de fidélité aux traditions : ainsi l'établissement situé à Celle de Cortona, plusieurs fois cité, est toujours désigné par le terme de locus (ou domus) alors qu'Élie a fait construire une église et un couvent (en ville) dès $1245^{43}$.

Mais souvent le mot est employé en concurrence avec conuentus pour beaucoup d'établissements franciscains, qui sont désignés par les deux termes tantôt dans des passages différents, tantôt dans des phrases successives. Sauf dans quelques cas comme ceux que nous avons mentionnés, il y a là un jeu stylistique évitant la répétition de conuentus, comme peut le faire également l'emploi de domus. Notons qu'alors locus ne paraît pas sensible à la répétition : ainsi p. 750 se succèdent six emplois de locus pour Reggio, pour lequel le terme de conuentus est employé ailleurs, ou p. 808/9 où il y a cinq emplois consécutifs, pour La Verna il est vrai ${ }^{44}$.

Pourtant, malgré l'impression que locus peut remplacer conuentus, les situations des deux termes ne sont pas symétriques au vu des constructions grammaticales dans lesquelles ils entrent. Par exemple le terme le plus large, locus, peut remplacer conuentus pour éviter des répétitions dans un enchaînement, mais on ne trouve guère conuentus après locus (à une exception près, où les deux termes sont assez éloignés). D'autre part locus est plus rarement déterminé par un élément géographique (14 emplois seulement construits directement sur le type locus Aluerne et 25 avec intercalée la mention fratrum (Minorum/Predicatorum) sur le type de locus fratrum Minorum de Parma, soit moins de $30 \%$ au total) et jamais par un adjectif marquant la localisation (au contraire des groupes du type conuentus Ianuensis), ce qui souligne la dissymétrie de fonctionnement des deux termes locus et conuentus: conuentus n'est pas pleinement entré dans l'emploi de « établissement ».

34 En outre les emplois au pluriel sont réservés à loca, à trois exceptions près que nous avons déjà rencontrées et qui seront reprises en conclusion ${ }^{45}$. De même, lorsqu'il s'agit d'évoquer le responsable d'un couvent, le guardianus, le terme est employé seul, avec le 
génitif du nom du lieu, ou dans le groupe guardianus loci/domus (7 emplois) dans 47 cas sur 49 ; on ne trouve que deux fois guardianus conuentus, un groupe encore visiblement tout à fait marginal.

Enfin dans deux domaines où l'aspect matériel est au premier plan, le terme de conuentus est totalement absent. Lorsqu'il s'agit de construction ou de destruction de bâtiments, on ne peut trouver que locus ou d'autres mots à valeur clairement matérielle : ainsi de la destruction par incendie d'un couvent de Prêcheurs (Combustus est locus fratrum Predicatorum in ciuitate Verone, p. 767, 24 et 25) ou de la Bibliothèque du couvent des Mineurs à Lyon (totum dormitorium cum libris omnibus est combustum, p. 768, 6) ; de même pour une construction, on trouve locus p. 902, 24, pro loco eorum edificando ou p. 708, 29 sq. la mention de maisons (domus) achetées à côté du locus des Mineurs pour s'agrandir, et sic amplificauerunt locum suum.

Quant aux mentions des sépultures dans les lieux dépendants des couvents de Mineurs - et l'on sait à quel point les franciscains tenaient à ce privilège qui leur a valu bien des jalousies !-, elles peuvent être faites in ecclesia ou in loco fratrum Minorum. On dénombre 59 évocations de sépultures, dont 36 (au total) chez les Mineurs : 23 sont dites in loco fratrum Minorum, pas une seule in conuentu, alors qu'on a par exemple chez les Clarisses ad monasterium illarum dominarum Ordinis sancte Clare iacet sepulta (p. 77, 21), ou chez les Bénédictins in monasterio Sancti Benedicti... in quo comitissa Matildis est sepulta (p. 78, 16).

Conclusion

La Chronique de Salimbene s'est donc révélée un bon terrain pour observer l'usage que font les Mineurs des termes dont ils ont hérité ou qu'ils sont en train de créer pour désigner leurs établissements. Dans ce domaine où les Mendiants ont joué un rôle central, où l'évolution sémantique de leur vocabulaire a suivi le rythme rapide du développement des Ordres Mendiants eux-mêmes, certains mots ont, quand écrit Salimbene, déjà perdu toute place importante dans le vocabulaire de l'Ordre ${ }^{46}$, d'autres sont très largement utilisés mais ne connaîtront pas toujours le même succès : locus, encore dominant chez Salimbene et après lui, cèdera du terrain (peut-être trop lié à la modestie de ses origines pour être toléré s'agissant de constructions qui n'ont plus guère de rapport avec les pauvres masures au toit de paille des débuts de l'époque de saint François) et ne laissera pas en français ${ }^{47}$ par exemple les mêmes descendants que domus, « maison (religieuse) », ou conuentus, « couvent ».

Conuentus est une "valeur montante » : terme d'apparition assez récente dans ce sens spécifique (mais qui avait pour lui l'avantage d'une existence ancienne, et fréquemment employée dans un contexte religieux), il n'a pas encore achevé son évolution chez Salimbene à la fin du XIII ${ }^{\mathrm{e}}$ siècle dans le vocabulaire des franciscains euxmêmes ${ }^{48}$, la valeur matérielle de "bâtiments conventuels " ayant bien déjà apparu, mais restant encore numériquement peu attestée. Quant à la tentative de 1260 pour introduire une terminologie précise, elle a visiblement fait long feu, et il est assez naturel que les historiens de l'Ordre ne la mentionnent en général même pas.

Toutefois l'évolution en cours permet à Salimbene d'utiliser habilement les arrièreplans de l'opposition conuentus/locus dans la rivalité avec les Prêcheurs, et c'est justement là qu'apparaît le plus clairement le sens matériel de conuentus. Il y en a trois exemples, dont j'ai déjà parlé plusieurs fois de façon allusive. Deux, déjà cités, doivent être remis dans leur contexte et $j$ 'en ajouterai un dernier ${ }^{49}$. 

Mineurs et Salimbene s'étonne que les Prêcheurs ne se soient pas installés dans cette ville ; la réponse, avec l'emploi de l'adjectif bonus qui s'oppose à l'évocation peu aimable de la multitude des petits établissements franciscains dit assez que, selon Salimbene, les Prêcheurs tiennent à leur confort ou à la démonstration de leur puissance : «Et comme je lui demandais pourquoi les frères prêcheurs n'avaient pas de couvent (conuentum) à Vienne, il me dit qu'ils préféraient avoir à Lyon un bon couvent (unum bonum conuentum) plutôt que d'avoir une telle foule d'établissements (locorum) $»^{50}$.

41 Un peu plus loin, en 339, 2, Salimbene évoque de nouveau la rivalité latente entre les deux Ordres : soulignant avec plaisir combien les habitants d'Hyères sont dévoués aux Frères Mineurs, et ce pour le plus grand bénéfice de leur âme, il en profite pour jeter une pierre dans le jardin des Prêcheurs qui ont dédaigné cette petite ville comme ils ont dédaigné Vienne! «Les habitants de cette ville éprouvent une grande dévotion pour les frères Mineurs et ils écoutent volontiers la parole de Dieu quand elle est prêchée par les frères. C'est que les frères prêcheurs ne sont pas établis à cet endroit parce qu'ils se plaisent davantage à habiter dans de grands couvents (in magnis conuentibus) que dans des petits et qu'ils en retirent davantage de soutien $»^{51}$. Ce n'est certes pas par hasard que l'auteur utilise le verbe predicare pour parler du soin que les Mineurs ont du salut de leurs ouailles; c'est certes une de leurs fonctions, mais le rapprochement avec Predicatores suggère que ces derniers ont failli à leur devoir. L'emploi de consolor (« encourager, rassurer, soutenir ») n'est guère plus aimable ! prêcheur, qui vient d'assister à une éblouissante démonstration de la supériorité d'Hugues de Digne. Le socius de frère Pierre s'informe de la qualité de l'orateur : est-il «prelatus, guardianus uel custos uel minister?». Mais l'humilité profonde de Hugues lui interdit de rechercher les charges: il ne veut recevoir aucune dignité, répond Salimbene ${ }^{52}$. Et le socius de louer ces éminentes qualités et d'enchaîner : "Mais je me demande avec étonnement pourquoi il n'habite pas dans de grands couvents (in magnis conuentibus) » ${ }^{53}$, ce qui permet à son interlocuteur «d'enfoncer le clou» : «Je lui ai dit : "C'est à cause de son humilité et de sa perfection, parce qu'il trouve davantage de soutien à être dans de tout petis établissements (in paruulis locis)" ${ }^{54}$. On aura remarqué que les verbes morari et consolari sont ceux-là mêmes de l'exemple précédent, auxquels il font écho. Rappelons enfin que juste avant ce dialogue, Hugues avait souligné la nécessité de donner aux Prêcheurs une bonne leçon pour leur fatuité et leur manque de reconnaissance pour l'accueil généreux qui leur est fait, puisque les Prêcheurs prétendaient que les Mineurs n'étaient que des ignorants stupides, surtout par comparaison avec la culture des dominicains: "Ces braves gens (c'est-à-dire: les Prêcheurs), dit Hugues, se vantent toujours de leur savoir et ils disent que c'est dans leur Ordre qu'on trouve la source de la sagesse... Ils disent aussi qu'ils sont passés chez des ignares, quand ils passent par les établissements des frères Mineurs, où on les sert avec amour et empressement. Mais par la grâce de Dieu, maintenant, ils ne pourront plus dire qu'ils sont passés chez des ignares $! »^{55}$.

Démonstration est faite, me semble-t-il, de la finesse avec laquelle Salimbene utilise toutes les ressources du latin, y compris les ressources nées d'une évolution toute récente. 


\section{NOTES}

1. Voir par exemple le " petit » Robert ou le Dictionnaire étymologique Larousse. Le Grand Robert de la langue française ( $2^{\mathrm{e}}$ édition) signale quant à lui le terme au début du XII ${ }^{e}$ siècle, sous la forme " convent ». Cf. également le Littré (avec des exemples d'emploi au XIII e siècle au sens de " union, société »).

2. On trouve par exemple au XI siècle, dans la Collectio canonum in V libris, 1, préf. III, 1. 25 : « Coetus uero conuentus est uel congregatio a coeundo id est conueniendo in unum, unde et conuentus est nuncupatus, quod ibi omnes conueniunt, sicut conuentus, coetus uel concilium a societate dicitur multorum in unum $»$. Une définition identique est reprise au XII ${ }^{\mathrm{e}}$ siècle par Hugues de Saint Victor dans son Didascalicon de studio legendi, 4, 87, 20.

3. Définitions du dictionnaire Robert.

4. Ce projet est celui du groupe de traduction d'œuvres latines médiévales dont je m'occupe avec Michèle Brossard-Dandré. La traduction intégrale de cette chronique, jamais publiée en français, devrait paraître d'ici quelques mois. Elle s'appuie sur le texte latin édité par G. Scalia, Salimbene de Adam, Cronica, 2 vol., Bari, 1966, repris par la nouvelle édition du même auteur dans la collection du Corpus Christianorum, Continuatio Mediaeualis, Turnhout, 1998-1999. La pagination de la première édition, que j'utilise ici, est reprise en marge dans la deuxième.

5. Pour l'emploi général de locus au sens d'établissement religieux (en dehors de toute référence franciscaine), voir l'article du Fr. M.-A. Dimier, « Le mot locus dans le sens de monastère », Revue Mabillon, 58, 1972, p. 133-154, qui souligne « le grand nombre de monastères de tous ordres, dans le vocable desquels le mot de locus ou de lieu entre en composition » (p. 134); toutefois les exemples cités ne sont pas tous convaincants et, dans bon nombre d'entre eux, le sens prétendu de monastère a chance de n'être qu'un effet de sens dû au contexte. Je remercie Éric Palazzo de m'avoir signalé cet article.

6. Par exemple chez M.-Th. Laureilhe, Jourdain de Giano, Thomas d'Eccleston et Salimbene de Adam, Sur les routes d'Europe au XIII 'e siècle, Paris, 1959.

7. Par exemple chez le P. Gratien, Histoire de la fondation et de l'évolution de l'Ordre des Frères Mineurs au XIII siècle, Paris-Gembloux, 1928. Nouvelle édition avec mise à jour bibliographique.

8. Ainsi chez O. Guyotjeannin, Salimbene de Adam, un chroniqueur franciscain, Turnhout, 1995, le groupe in loco fratrum Minorum est traduit par « chez les frères mineurs ».

9. Pour situer précisément de quelle époque il s'agit, rappelons que Salimbene est né à Parme en 1221, l'année de la mort de saint Dominique et cinq ans avant la mort de saint François. Il a reçu une bonne éducation avant de devenir franciscain ( $d$ 'où son usage efficace du latin, même s'il y mêle parfois de l'italien, très consciemment en général) ; il entre dans l'Ordre en 1238. Il écrit sa Chronique vers la fin de sa vie, entre 1283 et 1288, date à laquelle on perd toute trace de lui (il a 67 ans).

10. Il y a évidemment une exception pour parler de l'ermitage (eremus, eremitorium), qui face à l'habitation urbaine (celle qui nous intéresse ici) constitue l'autre pôle des installations franciscaines. Cf. par exemple G. G. Merlo, Tra eremo e città, Studi su Francesco d'Assisi e sul francescanesimo medievale, Assise, 1991.

11. Parmi de nombreux exemples possibles, voir la Regula non bullata (vers 1221): «Caueant sibi fratres ubicumque fuerint in eremis uel in aliis locis quod nullum locum 
sibi approprient nec alicui defendant $(7,13)[. .$.$] Et nullo modo fratres recipiant nec$ recipi faciant nec quaerant nec quaeri faciant ... denarios pro aliquibus domibus uel locis $(8,8)[. .$.$] Iniungo omnibus fratribus meis tam clericis quam laicis euntibus per$ mundum uel morantibus in locis quod... $(15,1) »$.

12. Sur les maisons où s'installent les franciscains à leurs débuts, voir J. Dalarun, « Les maisons des frères. Matériaux et symbolique des premiers couvents franciscains », dans Le village médiéval et son environnement. Études offertes à J-M. Pesez, Paris, 1999, p. 75-95: on y trouvera aussi le rappel de l'enjeu qui se cache dans le choix des mots pour désigner ces établissements, particulièrement $p$. 75-7. Sur ce sujet, il vaudrait la peine de poursuivre l'enquête en examinant le vocabulaire employé par d'autres Ordres dans des conditions comparables. Ainsi, pour les Camaldules, C. Caby, De l'érémitisme rural au monachisme urbain. Les Camaldules en Italie à la fin du Moyen Âge, Rome (BEFAR 305), 1999, signale que « de nombreuses communautés s'installent dans des édifices préexistants dont la structure ne subit que des retouches partielles et pas nécessairement au moment précis de l'installation des Camaldules »(p. 322). Voir le livre II, chapitre V, 1. Les bâtiments monastiques, p. 313 sq. : malheureusement cet ouvrage ne s'intéresse guère aux problèmes de vocabulaire et en particulier à la question qui m'occupe ici ; on notera toutefois l'apparition fréquente du terme monasterium dans les textes latins que les notes fournissent en abondance. Je remercie Nicole Bériou qui m'a suggéré ce rapprochement, en même temps que plusieurs références bibliographiques ou corrections pour l'ensemble de cet article.

13. Cf. P. Gratien, Histoire de la fondation..., op. cit., p. 172.

14. J. MOORMAN, A history of the franciscan Order, from its origins to the year 1517, Oxford, 1968, p. 149, souligne qu'il s'agit là d'une « innovation ».

15. Statuta Generalia, Narbonne, IX, 20 : « Conuentum autem dicimus, ubi XIII fratres et supra possint continue commorari. » Dans ce texte, l'emploi de l'adverbe relatif de lieu $u b i$ et du verbe commorari suggèrerait plutôt l'interprétation du terme comme «bâtiments conventuels », mais le sens de « communauté » n'est pas impossible. Pour des textes antérieurs, voir C. Cenci, «De fratrum minorum constitutionibus praenarbonensibus ", Archivum Franciscanum Historicum, 83, 1990, p. 50-95: on y trouve les emplois habituels de locus au singulier ou au pluriel (fratres loci en 6 et 26, guardianus loci en 25, in locis fratrum/in locis en 62, 69, 70, 86 et 87, loca singula en 11), une attestation de domus (domus parisiensis en 29) ; en revanche conuentus n'apparaît pas, sauf de façon indirecte en 11 dans le groupe in locis conuentualibus (qui sera repris dans le texte des constitutions de Narbonne).

16. P. Gratien, Histoire de la fondation..., op. cit., p. 157-8.

17. Jourdain de Giano, Chronica, éd. H. Boehmer, Collection d'études et de documents, t. VI, Paris, 1908, ch. 73, 60. Cette étude du vocabulaire chez Jourdain de Giano et Thomas d'Eccleston a été réalisée à partir de la saisie informatique de ces textes telle qu'on la trouve dans le corpus du clclt-3, les CD-Rom du cetedoc publiés à Louvain la Neuve.

18. ID., ch. 47,42 .

19. ThOMAS D'ECCLESTON, Tractatus de aduentu Fratrum Minorum in Angliam, éd. A.G. Little, $1951^{2}$ (Paris, 1909). On compte 13 emplois de locus avec un nom de lieu (en général le nom de la ville au génitif, rarement la construction avec de+ablatif) et une trentaine de locus sans complément pour préciser la localisation (il est souvent question d'ampliatio locorum).

20. Une vingtaine d'emplois de domus sur l'ensemble du traité en quinze chapitres. 
21. Pour évaluer la valeur comparative de ces chiffres, rappelons que la Chronique de Salimbene occupe plus de 900 pages dans l'édition Scalia. Toutefois, la proportion des attestations des termes étudiés ici sont révélatrices chez chaque auteur : voir ci-dessous pour locus et domus chez Salimbene.

22. Une telle étude du vocabulaire est rendue possible par le dépouillement informatique (lemmatisé) publié sur microfiches par le cetedoc avec la nouvelle édition de G. Scalia, 1998-1999, et par l'utilisation du corpus du clclt.

23. Sur les 376 apparitions de locus, $60 \%$ gardent le sens courant de « lieu, endroit». 24. On perçoit là chez Salimbene une forte revendication du terme face aux concurrents directs des franciscains que sont les Prêcheurs, comme nous le verrons mieux en conclusion.

25. Le terme de « clarisses » peut prêter à discussion puisqu'il n'apparait pas dès l'origine : on pourrait parler des «Pauvres Dames », «Pauvres Sœurs » ou " femmes de l'Ordre de sainte Claire "; cf. M. Sensi, « Clarisses entre Spirituels et Observants », dans Sainte Claire d'Assise et sa postérité. Actes du colloque organisé à l'occasion du VIII centenaire de la naissance de sainte Claire, UNESCO, 29 septembre- $-^{\text {er }}$ octobre 1994, éd. G. BrunelLobrichon et al., Nantes-Paris, 1995, p. 101-118 (voir par exemple p. 101). À cause de l'influence de la règle bénédictine, le terme habituel qui désigne les établissements de Clarisses est chez Salimbene monasterium (cf. P. Gratien, Histoire de la fondation..., op. cit., p. 596), employé 37 fois dans ce cadre sur les 98 attestations du mot ; on peut ajouter un exemple de plus, en 88,6 , où monasterium s'applique exceptionnellement à une maison abritant à la fois des Clarisses et des frères Mineurs, monastère d'ailleurs qualifié de " très riche ", ditissimum. L'ouvrage Sainte Claire d'Assise. Documents rassemblés par le P. Damien Vorreux, Paris, 1983, $2^{\mathrm{e}}$ éd. 2002 est entièrement en traduction et ne permet pas d'étudier le vocabulaire latin employé par les textes ici rassemblés.

26. Le terme habituel pour les Prêcheurs est locus ou domus, pour les autres ordres (Cisterciens, Bénédictins...) monasterium.

27. Cet emploi sera désormais exclu du décompte.

28. Le décompte n'est pas facile à faire dans le détail. Ainsi il me semble clair que la précision géographique est donnée dans la phrase en 307, $2:$ « Iui Altissiodorum et habitaui ibi, quia a ministro Francie de illo conuentu specialiter fueram factus »; la présence d'un démonstratif comme iste (ex. p. 472, 21 et 22) ou ille, après l'indication d'un lieu, me paraît jouer le même rôle que l'indication géographique. Mais en l'absence de cet élément, on peut discuter de l'analyse à faire. Il y a cependant encore des cas où la proximité de l'indication géographique et la propension du latin à ne pas exprimer un démonstratif s'il peut être déduit facilement du contexte me semblent autoriser à classer l'emploi dans la catégorie des emplois " géographiques », par exemple p. 305, 10, « quidam fratres Gallici de conuentu » renvoyant à la 1. 6, « habitaui in conuentu Senonensi ».

29. Notons au passage que, sur plus de 50 établissements franciscains cités chez Salimbene, les 26 qui sont exclusivement ou entre autres désignés par le terme de conuentus, sont de grands établissements, ce qui semble respecter la définition de 1260 . 30. Cependant le sens de " communauté » est tout à fait possible, cf. par exemple p. 323, 9 : « Cum autem uisitassem fratres de Altisiodoro, de quorum conuentu fueram, iui una die Vergeliacum ", ou encore p. 447, 2 ou 867, 23...

31. "Et ecclesia in solario erat ; et totus conuentus illarum dominarum numero LXX duarum congregatus ibidem » (p. 579, 35). 
32. "Item raro comedebat in conuentu cum aliis fratribus, sed semper seorsum in camera sua solus » (p. 231, 18).

33. "Ad episcopalem ecclesiam erat iturus; et totus conuentus congregatus uolebat eum associare... Volebant ipsum audire. Et dixit: "Non requiro talem honorem, quia non sum papa. Si autem uolunt audire, ueniant postquam fuerimus ibi, et ego precedam cum uno socio et non ibo cum ista caterua." Cum autem peruenerunt illuc, inuenerunt omnes congregatos et paratos ad audiendum. » On peut encore ajouter 494, 21, non presente conuentu ; 450, $1 ; 580,17$. L'emploi de conuentus dans le document cité par Salimbene en 907, 21 est aussi à ranger ici.

34. Signalons aussi que l'on trouve parmi ces quatre exemples les deux seuls emplois au pluriel, qui sont donc tout à fait marginaux.

35. "Si habebant summo mane in conuentu de mortuis aliquam missam, ex illa in aliquibus locis contenti erant et aliam... penitus dimittebant » (p. 439,6).

36. Ch. 22, 4 et ch. 50, 7.

37. À propos de cette discrétion de Salimbene sur la richesse de certains couvents, il est amusant de voir dans quel cadre il parle de cloître. On sait qu'une telle construction était aux yeux des premiers franciscains un symbole du luxe ostentatoire de certains monastères et qu'ils tenaient à tout prix à l'éviter. Deux anecdotes le disent clairement. Dans le Sacrum commercium de saint François et de dame Pauvreté, lorsque cette dernière a demandé à voir le cloître des frères, le saint et ses compagnons lui ont, du haut d'une colline, montré la campagne environnante en lui disant : « Voilà notre cloître ! ». De la même façon, Jourdain de Giano est scandalisé devant l'offre des bourgeois d'Erfurt qui lui proposent de construire un cloître à l'usage des Mineurs et répond : "Je ne sais pas ce que c'est qu'un cloître, bâtissez-nous seulement une maison près de la rivière de façon que nous puissions y descendre pour nous laver les pieds » (trad. de M.-Th. Laureilhe, ch. 43). On ne rencontre que deux mentions de cloître pour tous les couvents de Mineurs dont parle Salimbene. À Reggio, in conuentu Regino (p. 768, 11), le jour de la Toussaint, après matines, au sortir de l'église, Salimbene lui-même signale qu'il passe dans le cloître, mais c'est parce que tout naturellement, c'est le seul endroit pour observer un curieux phénomène céleste, une pluie par ciel serein. L'autre mention se place dans un récit aussi amusant qu'édifiant, à Tarascon, qualifié là encore de simple locus (p. 430,23) alors que l'auteur désigne ailleurs l'endroit comme un bonus conuentus : on a prévu pour le ministre général Jean de Parme un lit placé dans la pièce où sont aussi les lits des autres hôtes, mais il s'attarde dans ses prières et personne n'ose aller dormir avant lui, malgré la fatigue. Il faut que Salimbene aille solliciter sa permission pour que tous puissent aller se coucher. Lui cependant l'attend pour lui montrer le lit qui lui a été préparé, mais le ministre général refuse une couche qu'il estime trop confortable et oblige Salimbene à y coucher à sa place. Or ces longues prières se faisaient in claustro.

38. Rappelons qu'on appelle génitif subjectif la construction qui fait un complément de nom de ce qui serait le sujet dans une locution verbale. Conuentus fratrum équivaut à fratres conueniunt.

39. Cet emploi avec indication géographique me semble récent à en croire les sondages effectués d'après le clclt. Sur les 508 conuentu (à l'ablatif) repérables dans les auteurs médiévaux du corpus (CD-Rom II du clclt-3), on relève seulement une dizaine d'exemples avec adjectif d'origine ou génitif de nom de ville (y compris dans les sens non religieux) auxquels il faut ajouter les exemples franciscains (78 exemples chez 
Salimbene et 2 chez Thomas de Eccleston), soit 89 \% d'emplois de ce type au Moyen Âge chez les franciscains.

40. Locus et domus sont en particulier couramment employés pour les Prêcheurs.

41. J. Moorman, Medieval Franciscan Houses, New York, 1983.

42. Il y a ainsi plusieurs exemples d'installations franciscaines non répertoriées par J. Moorman.

43. Dans ce cas, le sens toujours perceptible du nom du lieu, « les cellules », a pu jouer un rôle. Notons de même qu'à Reggio, le premier établissement des frères Mineurs vendu aux Clarisses en 1256 quand les Mineurs se sont installés plus confortablement, est nommé locus uetus, p. 671, 25. À Assise encore, on mentionne un conuentus, mais s'il s'agit de parler de la sépulture de François, le terme est in loco Beati Francisci, p. 609, 13. 44. Les répétitions de conuentus sont plus rares, cf. p. 431/2, cinq emplois pour le couvent de Gênes, souvent cité, une seule fois sous le terme de locus.

45. Voir par exemple p. 318,8 ou $364,21$.

46. Ainsi area, l'emplacement pour bâtir, est absent chez Salimbene mais encore assez fréquent chez Thomas d'Eccleston où il entre en concurrence avec domus pour désigner les bâtiments eux-mêmes (une vingtaine d'emplois). Habitaculum, hospitium, deux fois présents chez Thomas d'Eccleston, n'ont pas une seule application aux Frères Mineurs chez Salimbene.

47. L'histoire de ce vocabulaire franciscain latin pourrait être complétée par l'étude de ses descendants dans plusieurs langues modernes, romanes ou non (italien convento, anglais convent...), ce que je n'ai pas eu le temps de faire.

48. Il serait intéressant de mener une enquête sur le vocabulaire utilisé à la même époque soit par des dominicains, soit par des écrivains extérieurs aux Ordres Mendiants. Pour compléter toutefois ce que mon enquête a forcément de partiel puisqu'elle ne s'intéresse guère qu'à un seul auteur parmi les franciscains, j'ai effectué un sondage sur des documents conservés dans un des couvents franciscains où a vécu Salimbene. J'ai utilisé l'ouvrage de V. Tirelli et M. Tirelli Carli, Le pergamene del convento di S. Francesco in Lucca (secc. XII-XIX), Rome, 1993 et dépouillé les documents pour les années 1220 à 1288, ce qui correspond en gros à la vie de Salimbene. Sur environ 90 documents répertoriés (numéros 13 à 100), 48 des documents dont le texte est donné concernent les Mineurs de Lucques ou l'Ordre en général. Les termes utilisés pour localiser les établissements des Mineurs sont très souvent ecclesia (l'église de sainte Marie-Madeleine est entre les mains des franciscains), parfois domus ou locus, mais aussi terra ou oratorium dans les actes les plus anciens. Le terme de conuentus apparaît pour la première fois dans un acte de 1252 (document 30) mais l'expression ne permet pas de préciser son sens exact : « conuentus Sancte Marie Maddalene fratrum Minorum de Luca ». Au total le terme n'est utilisé que dans 14 actes pour parler des Mineurs et dans 4 autres pour des moniales ou d'autres Ordres. Le contexte n'est souvent pas assez éclairant pour nous renseigner sur la valeur, déjà matérielle ou non, du mot : on notera toutefois plusieurs emplois de locutions comme « pro eisdem abbatissa sororibus et monasterio et conuentu », ou « abbatisse et conuentui monasterii de Gactaiola ordinis Sancti Damiani » (document 40) où la présence de monasterium laisse entendre que conuentus doit être pris dans son sens étymologique. De même le redoublement de termes quasi-synonymes - fréquent dans les actes juridiques - me semble apparaître dans une expression comme pro collegio et conuentu ipsorum fratrum (document 33). En conclusion, les actes conservés du couvent de Lucca montrent une utilisation restreinte du terme de conuentus à partir du milieu du xiii ${ }^{\mathrm{e}}$ siècle, principalement dans les actes 
rédigés à Lucques même, mais une fois au moins dans un acte de la chancellerie pontificale (document 39, de 1258 : « dilectorum filiorum fratrum Minorum in conuentu lucano ») ; la spécificité du corpus ne permet pas de dire si le terme est employé consciemment en fonction de la définition donnée à Narbonne en 1260, il reste en tout cas peu utilisé dans l'échantillon analysé.

49. On remarquera que l'on trouve ici deux des trois emplois au pluriel de conuentus, et de surcroît les deux seuls emplois pluriels qui soient bien de la main de Salimbene (puisque le troisième est en fait dans un document cité p. 907, 24) ce qui souligne un emploi tout particulier.

50. "Cumque interrogassem quare in Vienna fratres Predicatores conuentum non habebant, dixit michi quod potius uolebant Lugduni unum bonum conuentum habere quam uellent habere tantam locorum multitudinem ».

51. «Hi fratribus Minoribus ualde deuoti sunt et libenter audiunt uerbum Dei, cum predicatur a fratribus. Nam fratres Predicatores ibi locum non habent quia delectantur et consolantur in magnis conuentibus habitare potius quam in paruis ».

52. "Nullam prelationem habet, quia nullam habere uult ».

53. "Sed miror quare in magnis conuentibus non moratur".

54. "Cui dixi : "Propter humilitatem suam et sanctitatem, quia plus consolatur esse in paruulis locis" ".

55. « Isti boni homines (= Predicatores) semper de scientia gloriantur et dicunt quod in Ordine eorum fons sapientiae inuenitur... Dicunt etiam quod transierunt per homines ydiotas, quando transeunt per loca fratrum Minorum, in quibus eis caritatiue et sedule ministratur. Sed per Dei gratiam modo non poterunt dicere quod per homines ydiotas transierint... » (p. 364, 17-24).

\section{RÉSUMÉS}

Pour désigner les endroits où ils s'installent, les franciscains utilisent en latin plusieurs termes, en particulier locus et conuentus. De ce dernier terme est issu entre autres le français « couvent » (souvent utilisé pour traduire le mot latin), qui désigne d'abord les bâtiments où des religieux vivent en commun, puis l'ensemble des religieux qui composent la communauté. L'analyse des emplois de conuentus dans la Chronique du franciscain Salimbene de Adam permet de préciser une étape de l'évolution sémantique de ce mot et montre l'émergence du sens matériel : en cette fin $\mathrm{du} \mathrm{XIII}^{\mathrm{e}}$ siècle, conuentus désigne encore majoritairement la communauté des frères, conformément à l'étymologie du terme, mais le chroniqueur, dans un petit nombre de cas, use du mot pour désigner les bâtiments, non sans être conscient de certaines connotations attachées au choix des mots locus et conuentus.

Locus and Conuentus: Latin Vocabulary of Grey Friars Places by Salimbene de Adam. Several latin words are used by the grey friars to designate places where they dwell, among them locus and conuentus. From the latter comes the french word « couvent" (often used to translate the latin vocable), that designates the buildings where friars live together, and then the community of friars itself. A study of the term conuentus in the Chronicle of the grey friar Salimbene de Adam indicates a step in the semantic evolution of this word. At the end of 13th century, the word 
conuentus still designates essentially the community of the friars, according to the etymology, but Salimbene in some rare cases uses this word to designate the buildings. However, he remains aware of the backgrounds of both locus and conuentus.

INDEX

Mots-clés : franciscains, conuentus/couvent, évolution sémantique

Keywords : Salimbene de Adam, locus, franciscans, conuentus/french « couvent », semantic evolution

\section{AUTEUR}

\section{GISÈLE BESSON}

École Normale Supérieure des Lettres et sciences humaines, Département des Lettres, 15, parvis René Descartes, Boîte postale 7000, F-69342 Lyon Cedex 07 